\title{
URINARY DISORDERS OGCURRING IN THE NEONATAL PERIOD
}

\author{
W. S. CRAIG, B.Sc., M.D., M.R.C.P.ED.
}

(From the University Department of Child Life and Health, the Royal Maternity Hospital and the Western General Hospital, Edinburgh.)

Urinary conditions with a distinctive clinical picture are not uncommon in newborn infants. The majority of infants show excellent response to treatment and early diagnosis is of great importance. In any circumstances it is difficult to obtain specimens of urine from these small subjects and the difficulties are frequently increased as a result of the anuria which is a common accompaniment of urinary disorders in its early stages. Usually the characteristic clinical features of these cases allow of a diagnosis being made and treatment being begun before examination of the urine has been possible.

It is the purpose of this paper to give an account of observations made on a series of sixty-one cases collected over a period of three years. Details are given of urinary findings in these cases as well as in a series of healthy newborn infants, clinical signs and symptoms are described, treatment is indicated and the results recorded. Autopsy findings are given in connection with certain of the fatal cases and details of progress after discharge from hospital in children who recovered.

\section{Material.}

Specimens of urine were obtained from newborn infants in the Royal Maternity Hospital and the Western General Hospital, Edinburgh. A large number were from healthy children and provided a control series. Of the sixty-one children with urinary infection fifty-two were born in the former and nine in the latter hospital: all were under observation from the time of birth. Twelve children included in this series attended the Infant Clinic in connection with the Royal Maternity Hospital and were examined at intervals for a period varying from seven months to two years. Of those not regularly attending the clinic twelve reported by request, fifteen were visited in their homes and replies to a questionnaire were received in connection with nine others. Four children could not be traced: nine died while still in hospital.

Method of examination of the urine.-Specimens of urine from female cases were obtained by catheter: in male infants they were collected in test-tubes attached to the penis unless required for bacteriological investigation when catheter specimens were employed. Microscopical examination of the urine was carried out as soon as possible after receipt of the specimen, which was thoroughly stirred but not centrifugalized. The high-power lens was employed throughout and the findings recorded in not fewer than fifteen fields in connection with each specimen. This method of examination was along lines suggested by $\mathrm{Still}^{14}$ and Thomson ${ }^{15}$. 


\section{The urine of the healthy newborn infant.}

The urine of the newborn child differs from that of older subjects in that it may contain casts and cellular elements in the absence of disease in addition to certain amorphous and crystalline deposits ${ }^{12}$. In order to determine the normal microscopical findings in urine from newborn infants over two hundred specimens were obtained from healthy children whose ages ranged from twelve hours to fourteen days. Babies were selected who had been born spontaneously at term, had normal temperatures and were free from infection or disease as indicated by physical examination.

There were no cellular or other solid elements in one hundred-andsixty-three of the specimens: cells or casts or crystals or various combinations of the three were found in the remaining forty-eight, all of which were obtained from children aged four days or less. Epithelial cells were the most frequent finding, varying in number from three to four in a field to one in fifteen to thirty fields: they were most numerous in specimens from female cases. Casts were found in twelve specimens from children less than three days old: they were never more frequent than one in ten fields. Ten specimens contained polymorphonuclear leucocytes: they never occurred in greater number than one in ten to fifteen fields. Isolated red blood cells were seen in occasional specimens. Amorphous urates and crystals of uric acid or oxalate crystals were present in the majority of urines obtained during the first few days of life: quantitative estimation of these was not possible but in none of the cases from which they were obtained were there granular deposits on the napkins.

Forty-three catheter specimens were examined bacteriologically. Thirty-three were sterile, seven gave isolated discrete colonies of b. coli on culture, in the remaining three sparse growths were obtained, in two of staphylococcus albus and in one of a non-haemolytic streptococcus.

\section{Urinary findings in the present series.}

The sixty-one cases included in this series presented characteristic clinical features described in detail later, which led to examination of the urine. The urines all differed from those examined in the preliminary investigation and the microscopical abnormalities fell into one of the following groups:-

(1) Numerous pus cells (fig. 1). In forty-eight cases of the series pus cells were present in each field in numbers varying from five to one hundred and forty: clumping of the cells was noted in a number of the specimens. Hyaline and granular casts (one to four per high-power field) were present in thirteen of these cases.

Discussing pyelitis in the newborn Sauer ${ }^{13}$, Helmholz ${ }^{5}$, Conrad ${ }^{2}$ and others base their diagnosis on microscopical evidence of pus cells in the urine but do not indicate their frequency. Hoppe ${ }^{8}$ points out that slight pyuria may be found in healthy children in the neonatal period but considers that the presence of more than five pus cells per low-power field 
of an uncentrifuged specimen points to ' infection somewhere in the urinary tract.' Conrad ${ }^{2}$ and Hoppe ${ }^{\mathrm{s}}$ mention the occurrence of clumping of cells and the latter considers that it is characteristic of infection.

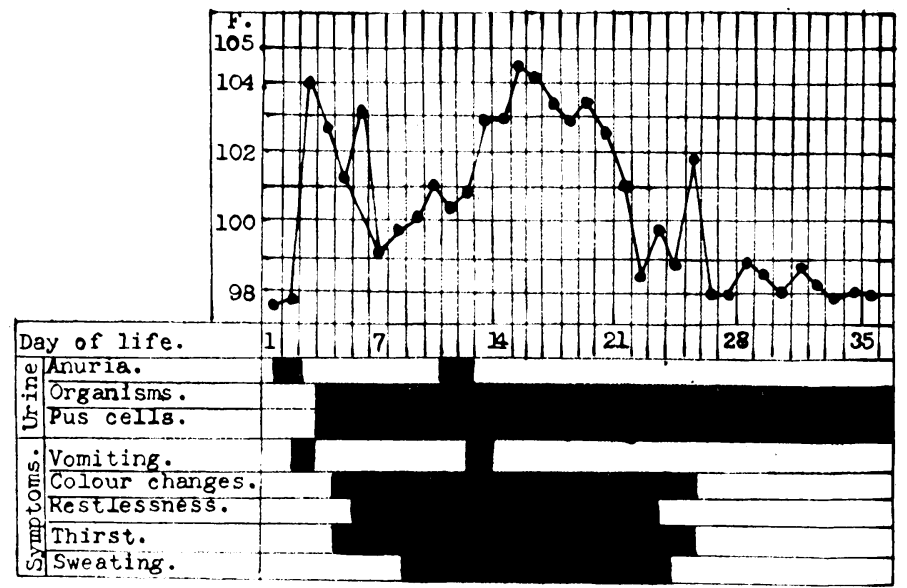

Fici, 1.-Male. Pyuria and bacilluria. Prolonged febrile course. Irine contained 15-30 pus cells per high-power field and was heavily infected with b. coli. Complete recovery: no history of recurrence of urinary symptoms at age 2 years 3 months.

(2) Organisms in the absence of pus cells. (fig. 2). Organisms were demonstrated in specimens of urine from three cases in the absence of pus cells. All three children were seriously ill, ran a high temperature and showed severe symptoms. The organisms could be seen in great numbers under the microscope and grew vigorously on culture: non-haemolytic streptococei and b. coli were cultured in one and pure growths of b. coli in two cases. Fresh specimens were examined from each case at intervals of one or two days.

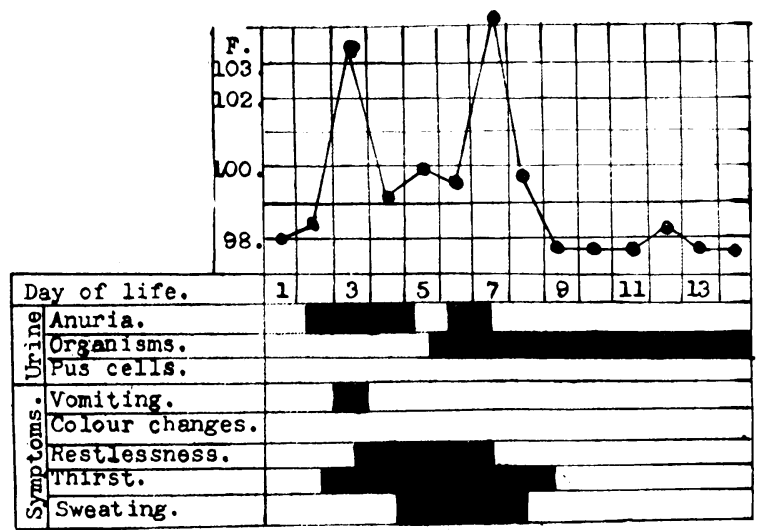

FIg. 2.-Male. Bacilluria. Catheter specimens of urine on 5 th, 7 th, 8 th, 11 th, and 14 th days of life contained no pus cells but gave a profuse growth of $b$. coli on culture. Complete recovery : no history of recurrence of urinary symptoms at age 14 months. 
Hoppe $^{8}$ emphasizes the need for repeated examination of the urine as cven in the presence of infection pus cells may be absent in occasional specimens. In each of the above cases specimens were examined at intervals of twenty-four to forty-eight hours on at least four occasions. Helmholz and Millikin ${ }^{6}$ consider that the urine of normal infants may contain a small number of organisms but they admit of the difficulty in excluding contamination. Ramsay ${ }^{11}$ is of the opinion that in many acute urinary infections the urine contains epithelial cells and colon bacilli in greatly increased numbers but no pus cells.

The findings in connection with the bacteriology of the urine of healthy children agree with those of Helmholz. In the three cases referred to above massive infection limited to the urinary tract was associated with severe symptoms similar to those occurring in cases of established pyelonephritis: in this respect and in the absence of pyuria these cases resembled a number described by Ramsay ${ }^{11}$. This writer mentions b. coli as the infecting organism: in one of the present cases both streptococci and b. coli were present.

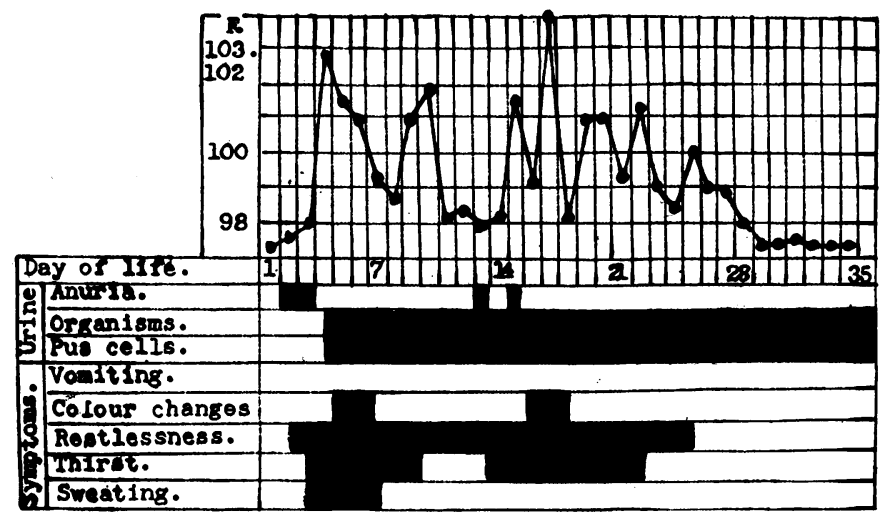

Fig. 3.-Female. Pyuria with mixed infection. Pus cells (5-15 per high-power field) present in all of nine specimens examined: on culture catheter specimens gave haemolytic streptococci and b. coli on the 5th, 18th, and 22 nd, days, and b. coli only on the 27th and 35th day. Complete recovery : no history of recurrence of urinary symptoms at age 11 months.

(3) Pyuria with mixed infection (fig. 3) was found in seven cases. B. coli were present in all seven: in four the accompanying organisms were non-haemolytic streptococci, in one b. pyocyaneous and in another haemolytic streptococci. In the seventh case specimens were obtained each alternate day: non-haemolytic streptococci and b. proteus were present in the first two: pus was not found until the third and b. coli were first cultured from the fifth specimen.

It is recognized that coliform infection of the urinary tract is the most common form: Thomson ${ }^{15}$ however draws attention to the variety of organisms which may be found and $\mathrm{Graham}^{3}$ considers that there is not such a predominance of coliform organisms in the newborn as in older children. The occurrence of mixed infection is not stressed in the literature but Helmholz" records a case in which the urine was infected by staphylococci. 
(4) Uric acid deposits in the absence of pus cells (fig. 4). A heavy uric acid deposit on the napkin occurred in association with pyuria in fifteen cases. In five cases similar deposits were noted in the absence of pus cells in the urine: casts were present (one to four per high-power field) in four of these five cases. Culture of the urine gave a profuse growth of b. coli in each instance.

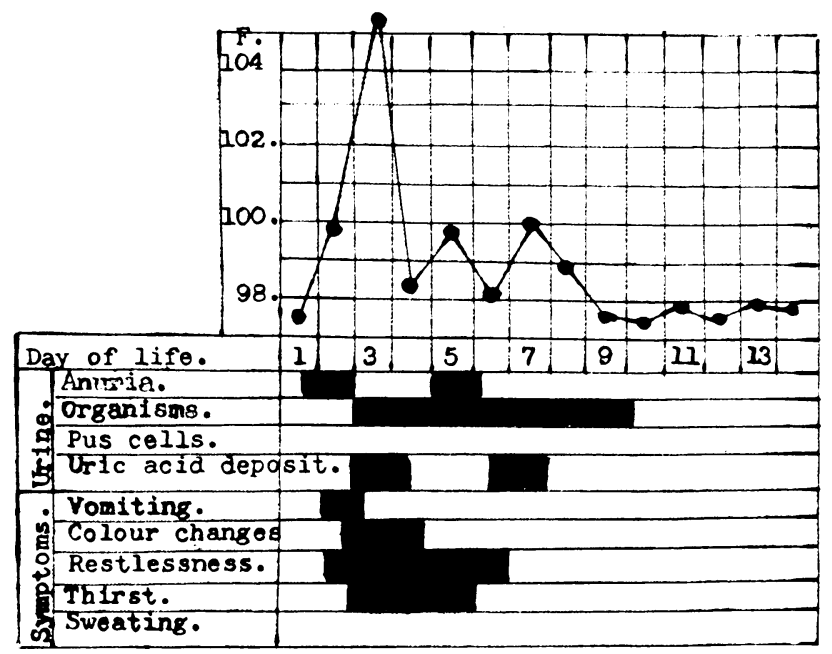

FIc. 4.--Female. Bacilluria with excessive uric acid crystal content of urine. Specimens of urine on the 3rd, 6th, 11th, and 1th days of life contained no pus cells. Short course, early disappearance of organisms. Complete recovery : no history of recurrence of urinary symptoms at age 18 months.

The clinical picture.

Table 1 gives a list of the elinical features met with in the present series and indicates the frequency with which they occurred. Individual signs

\section{TABLE 1.}

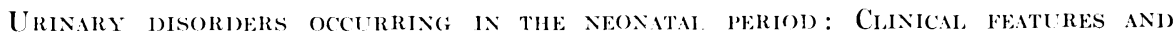
THEIR FREQUENCY IN A SERIES OF 61 cASES.

Clinical peatlues. $\quad$ No. Pases.

$\begin{array}{lllllllllllll}\text { Fever } & \ldots & \ldots & \ldots & \ldots & \ldots & \ldots & \ldots & \ldots & \ldots & 49 & 80\end{array}$

ANURIA OR MARKEI DIMINUTION IN URINARY OUTPLT...

$\begin{array}{lllllllllllll}\text { Thinst } & \ldots & \ldots & \ldots & \ldots & \ldots & \ldots & \ldots & \ldots & \ldots & 43 & \boldsymbol{7 0}\end{array}$

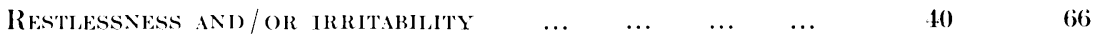

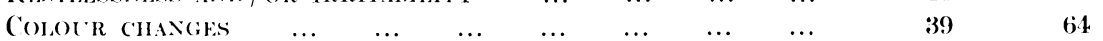

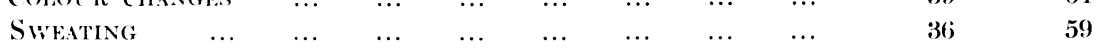

$\begin{array}{llllllllllll}\text { Vomitixg } & \ldots & \ldots & \ldots & \ldots & \ldots & \ldots & \ldots & \ldots & 31 & 51\end{array}$

$\begin{array}{lllllllllll}\text { Collapse } & \ldots & \ldots & \ldots & \ldots & \ldots & \ldots & \ldots & \ldots & 12 & 20\end{array}$

$\begin{array}{llllllllllll}\text { OЕIDEMA } & \ldots & \ldots & \ldots & \ldots & \ldots & \ldots & \ldots & \ldots & \ldots & 12 & 20\end{array}$

CONVULSIONS

Assochaten (ONHITONS :

A. INTESTINAL IRRITABILTTY $\quad \ldots \quad \ldots \quad \ldots$

b. Postratal intracranial nistress $10^{*} \quad 16$

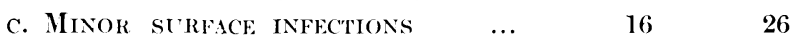

* The urinary condition occurred as a late complication in 9 of these cases. 
and symptoms were absent in certain cases but never to such an extent as to result in the loss of its characteristic nature by the clinical picture. The usual finding was one of a slight febrile irregularity commencing about the second or third day of life in association with occasional vomiting or anuria or both. Subsequent symptoms and signs were usually more pronounced and took the form of great restlessness and thirst accompanied by profuse sweating. A few cases ran an afebrile course but in the majority the temperature was characteristically irregular and tended to be high on occasion. Colour changes were common. Sudden collapse occurred occasionally and convulsions rarely: oedema was sometimes a feature.

(1) Onset. Symptoms of great severity frequently occurred with startling suddenness though rarely as the first indications of disease. The onset was sudden in only nine cases in the series : in all the remainder initial signs were vague in character and gradual in development. Litchfield and Gillman $^{9}$ and Sauer ${ }^{13}$ refer to the suddenness of onset as a characteristic of urinary infection in the newborn but other writers emphasize the variability of early symptoms.

Fever was the most common first symptom. In three cases described by Helmholz ${ }^{5}$ fever was the only symptom and elevation of temperature was the first evidence of illness in six cases recorded by Graham $^{3}$ and two by Paterson $^{10}$.

Vomiting and diminution in urinary output were the symptoms next in order of frequency: Sauer ${ }^{13}$, Litchfield and Gillman ${ }^{9}$ consider that gastrointestinal symptoms are usually the first evidence of infection and in two of three cases described by Conrad ${ }^{2}$ diminished urinary excretion was the first feature.

(2) The amount of urine. Failure to pass urine either with normal frequency or in normal amounts was noted in the majority of the cases: this invariably occurred in the earlier days of the illness of which it was frequently the first evidence. It was not uncommon for the napkin to be merely moistened only once in the twenty-four hours and in several instances the interval was thirty-six hours or more. During the period of anuria there was no distension of the bladder and on the few occasions when the catheter was passed at the time, urine was not obtained. Later as diuresis was promoted polyuria was evident and persisted in convalescence, varying with the fluid intake. In fatal cases anuria was a striking feature during the terminal stages.

Von Reuss ${ }^{12}$ draws attention to the limited urinary output during the first few days of life: he quotes Kotscharowski as finding thirty-four per cent. of newborn infants failing to pass urine in the first twenty-four hours but he himself considers that in health, micturition usually occurs two or three times during each of the first two days and increases in frequency until the end of the first week. There is little reference in the literature to the output of urine in the presence of infection during the neonatal period but in two cases of ' congenitally acquired pyelitis ' ${ }^{\prime}$ Conrad ${ }^{2}$ noted that severe symptoms were preceded by the passage of minute quantities of urine at lengthy intervals. The picture he describes closely resembles that found in a large number of the present series of cases. 
(*) Fever. A rise of temperature was the most common initial sign: this was often only slight $\left(99-100^{\circ}\right.$ F.) but, more characteristic than the height of fever was the persistence of an irregular temperature. The temperature varied taken at four-hourly intervals and showed a suggestive irregularity when charted only once daily (see fig. 4). The highest temperature recorded was $108^{\circ} \mathbf{F}$. in a child who recovered: temperatures of $102^{\circ} \mathbf{F}$. or over occurred in fifty per cent. of cases. The severity of the fever was not related to the duration of the illness: in the relatively few cases where onset of the illness was sudden and of a grave nature hyperpyrexia was present from the outset.

Fever was absent in eleven surviving and one fatal case (fig. 5): in all of these, abnormal clinical features were present and the urine contained pus and organisms. The apyrexial cases occurred in full-time and

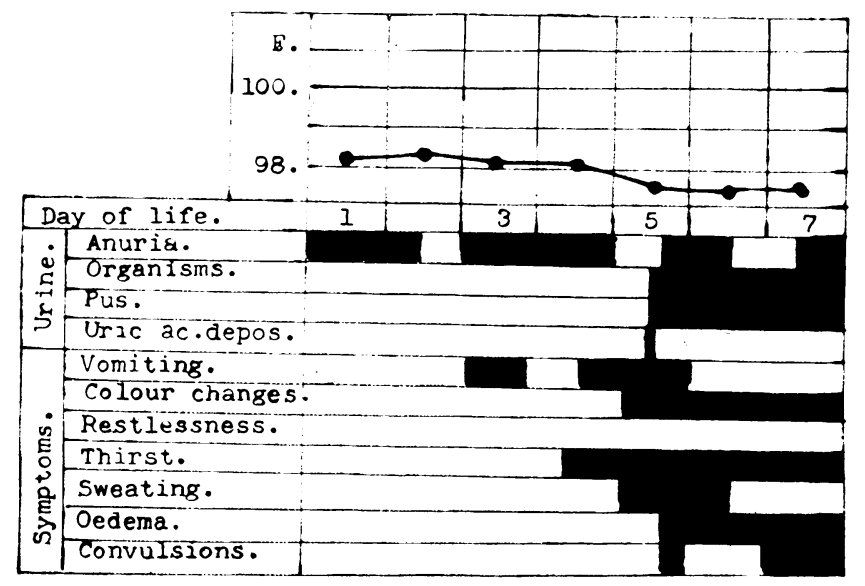

Fic, 5.-Female. Fatal case. Short afebrile course, characterized by only occasional passage of small amounts of urine, pyuria (54 pus cells per high-power field), terminal oedema and convulsions. Case 46.

premature infants: they tended to run a short course but in several instances the clinical features persisted for seven to ten days and the pyuria for several weeks.

Many workers consider pyrexia to be characteristic of urinary infection in the nowborn and it is mentioned in connection with the majority of recorded cases. Hoppe" and Sauer's state that it is ' usually present ' but attention has not been drawn to those cases in which there is no fever. There is a divergence of opinion as to the height of fever usually present. Litchfield and Gillman" consider that it seldom exceeds $101^{\circ}$ F.: Sauer ${ }^{1: "}$ is in agreement although a temperature of $102 \cdot 5^{\circ} \mathrm{F}$. occurred in one, and of $104^{\circ} \mathbf{F}$. in three of his series of fifteen cases: Ramsay" believes that high fever is characteristic. Cases described in the literature by Graham", Conrad", Paterson 11 and others indicate that temperatures of over $102^{\circ} \mathbf{F}$. occur in about half their number: this is in agreement with the present findings. 
(4) Vomiting occurred in fifty per cent. of cases and was not related to the severity of the other symptoms: it was absent in a number of the most severe cases. It took place during the early days of the illness and not infrequently was the first sign of disturbed health: it was rarely forcible and although occurring after every feed in a few instances usually took place only once or twice in the twenty-four hours. A striking feature was the cessation of vomiting which followed satisfactory diuresis even when other symptoms persisted for a longer period.

Of the twenty-eight cases recorded by Gueriot ${ }^{4}$, Graham $^{3}$, Conrad $^{2}$, Sauer ${ }^{13}$, Litchfield ${ }^{9}$ and Paterson ${ }^{10}$ vomiting was noted in thirteen. The nature of the vomiting was varied: in the majority it was only occasional, in one case it occurred after every feed and in another was projectile.

(5) Colour changes and collapse. Colour changes were characteristic: insidiously the face took on a dusky, grey appearance, not infrequently associated with a faint peri-oral cyanosis and a curious pallor of the scalp and forehead. At the same time a general limpness developed. This picture passed off as gradually as it developed, but while present, provided a striking contrast with the flushed skin, restlessness and irritability of the children at other times. In some cases changes of the nature described took place only on isolated occasions: in others they occurred frequently throughout the day during the height of the illness. They rarely occurred in the absence of fever and were noted in all fatal cases.

In a few cases the changes were more severe: there was sudden complete collapse, the skin was blanched, respirations were rapid and shallow, and the eyes were ringed and sunken. There was flaccid immobility of the trunk and limbs and a fixed expression of anxiety.

Thornson ${ }^{15}$ and Still ${ }^{14}$ describe attacks of collapse accompanied by colour changes and unexplained fever in older children as being suggestive of pyelitis. Dealing with the newborn infant Sauer ${ }^{13}$, Conrad ${ }^{2}$, Litchfield ${ }^{9}$ and others rerrark upon the pallor and anxious faces sometimes met with in the presence of urinary infection.

(6) Restlessness and irritability were characteristic of a large number of the children in the series. The restlessness was continuous over long periods and consisted of impetuous movements of head and limbs and ' squirming' of the body: in certain cases it developed into an actual fury, the child snatching at, and chewing, the bed covers. Restlessness frequently preceded elevation of temperatures: it was also met with in the absence of fever. It lessened in severity as the illness ran its course, often disappearing with surprising suddenness. As restlessness diminished irritability became more pronounced: unaccustomed to rationing of fluids during the height of the illness these children resented discipline and their cry of thirst became an imperious bellow. In contrast with the more usual state of agitation a few cases were limp and inert: the course run by these infants was never stormy, tended to be protracted, and was not characterized by high fever. 
Still ${ }^{14}$ draws attention to the extreme restlessness and misery found in older children suffering from pyelitis and Graham ${ }^{3}$ stresses their importance in the diagnosis of the condition in the newborn: both writers mention drowsiness and lethargy as occasionally occurring and Still considers that they point to a severe infection.

(7) Thirst was a feature of the majority of cases: it was always intense and in their desperation for fluids the children impetuously chewed their teat or 'gulped' down their fluids. Thirst was noted in the absence of fever but more commonly its appearance coincided with the initial rise of temperature; it was a constant accompaniment of fever but persisted for a considerable time after final settlement of the temperature.

(8) Sweating did not occur in the absence of fever and was usually accompanied by thirst: it was not affected by variations in the urinary output. Sweating usually occurred at the height of the illness: it was always profuse and the presence of beads of perspiration standing out on the forehead and scalp was characteristic of a number of cases.

(9) Oedema. Urinary abnormalities were found in only a small proportion of newborn infants showing oedema, and oedema was present in only twelve of the sixty-one cases of the present series. It was invariably preceded by marked diminution of urinary output and was present before death in five of the nine fatal cases. It occurred in one of the cases with severe urinary infection but no detectable pyuria, and in four cases where there was a heavy napkin deposit of uric acid and coliform infection of the urine but no pyuria. In males oedema first appeared in the suprapubic region and in females in the labia : the lower limbs were the next commonest site and in the most severe cases it was also found in the lumbar region, around the eyes and on the dorsal aspect of the hands. Appearing somewhat suddenly, oedema gradually disappeared following diuresis.

(10) Convulsions were uncommon but of grave significance. In five of six cases in which they occurred death followed the first fit within twentyfour to forty-eight hours. The fits in these cases were of a violent nature, involved the entire body, were accompanied by temporary unconsciousness and took place during periods of prolonged anuria. In the sixth case the infant was two days old and had not passed urine since birth when sudden, generalized spasticity was followed by slight clonic movements of the hands and face: a specimen of urine obtained a few hours later contained large numbers of pus cells. The child eventually recovered.

Thomson ${ }^{15}$ and Holt $^{7}$ refer to the relatively rare occurrence of convulsions in children suffering from pyelitis: Thomson found that they were more prone to occur in young babies and Holt that they were associated with severe infection. Dealing with urinary infection in the new-born Sauer ${ }^{13}$ mentions convulsions as one of the less common findings: they occurred in two of three cases recorded by Conrad $^{2}$ and in one of them were related to periods of diminished urinary output.

(11) Remissions occurred in a limited number of cases but only in one instance on more than one occasion: they occurred with the same frequency in the two sexes. Of more frequent occurrence were dcceptive periods when symptoms improved and the temperature settled temporarily, only to 
become worse again after noticeable diminution in the urinary output (see lig. 3). No recurrence of symptoms occurred in any of the children followed up alter discharge from hospital.

Holt ${ }^{i}$. Thomson ${ }^{1:}$ and others remark upon the frequency of relapse in cases of urinary infection in children. Holt states that fever may be interrupted by periods of normal temperature lasting several days and he considers that recurrence of symptoms may take place a few weeks to several vears after the initial illness. Return of fever and symptoms after the condition had apparently settled was noted in two cases of neonatal pyelitis by Graham": he also followed up two eases, one for a period of four months and the other for six months but in neither was there a relapse.

Associated conditions.- 1. Intestinal irritability. In ten cases of the series frequent, green foetid, motions containing a large amount of mucus were passed; in six the presence of abnormal motions was first noted two to three days after the detection of pyuria; in two the alimentary disturbance preceded the urinary condition by forty-eight hours and in the remaining two upset of urinary and bowel function occurred simultaneously.

Discussing pyelitis in children Thomson ${ }^{1.5}$, Still ${ }^{1.4}$, and Holt ${ }^{\tau}$, draw attention to the fact that the condition may either follow or accompany wastro-intestinal symptoms. No mention is made of the stools in a number of the recorded cases of neonatal pyelitis but while Litchfield" and Sauer." are of the opinion that gastro-intestinal symptoms are common and usually appear before the pyelitis is discovered Graham:" emphasizes the atsence of diarrhoea in all of the six cases described by him.

2 . Postnatal intracranial distress. Urinary infection occurred as a late complication in nine cases which had shown symptoms of cerebral hacmorrhage following delivery (fig. 6). Specimens of urine obtained during

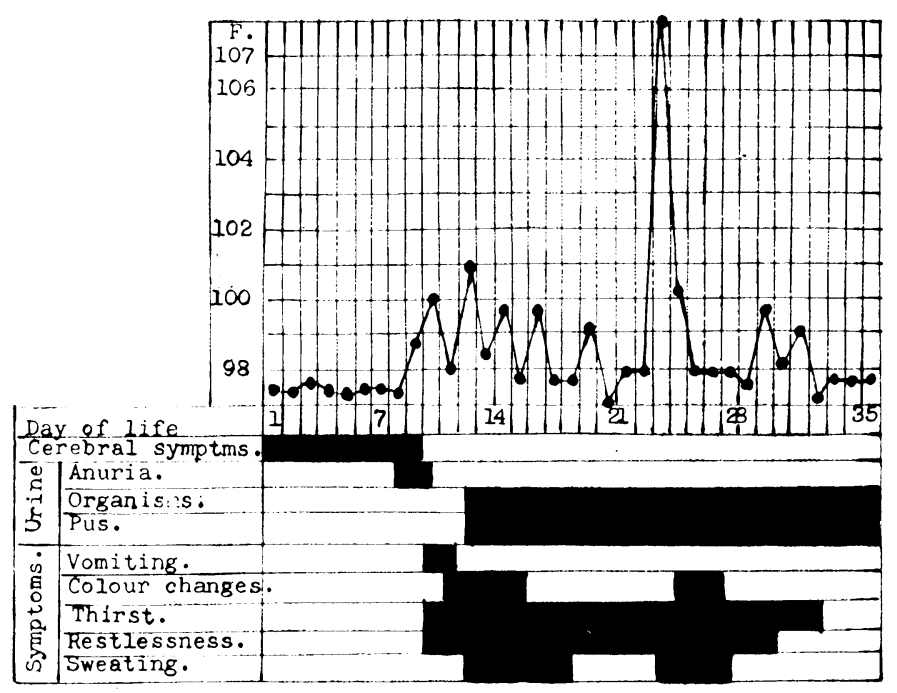

FI(i. 6.--Female. Pyuria and bacilluria as a complication of intracranial birth trauma. Specimens of urine on the the and th days of life contained no abnormalities and were sterile on culture: b. coli and pus cells (8-14 per high-power field) present in sperimens on the 10th, 16th, 25 th, and 34th days. Complete recovery: no history of recurrence of urinary symptoms at age of 1 year 10 months. 
the period of intracranial distress were normal: pyuria or bacilluria or both first occurred from the sixth to the eleventh day of life. Apart from the late development, the urinary condition ran a course similar to that of other cases in the series both with respect to symptoms and variability of duration. One of the nine children died and details of post-mortem findings are given on page 348 (case 14).

3. Minor surface infections. Weakened resistence to infection was evident in a number of patients whose illness had run a prolonged course. In these children sweating gave rise to a papular rash which frequently became pustular: the nose, eyes, and mouth were other common sites of low-grade infections.

Duration of the illness.- In almost every case abnormalites persisted in the urine for a varying period after the disappearance of symptoms and a number of children were discharged from hospital before the urine had become normal. Severe symptoms persisted from a few days to six weeks: they were not inevitably associated with a prolonged course, but symptoms persisting over a number of weeks were always severe. The duration of the illness was not related to the sex of the individual: in cases where mixed or unusually heavy b. coli infection was found the illness was severe and protracted. Cases in which the symptoms were persistent were also the cases in which bacteruria or pyuria remained longest after the disappearance of symptoms: several children had pus in their urine for seven or eight weeks after an apparently complete clinical recovery from a critical illness.

According to $\mathrm{Holt}^{7}$ the duration of an attack of acute pyelitis may vary from a few days to six or eight weeks and pyuria may persist for a much longer time. Recorded cases indicate that Holt's views apply equally well to the neonatal as to any other period in childhood: Litchfield ${ }^{9}$ and Sauer ${ }^{13}$ both give six weeks as the usual duration of illness in the newborn but Graham $^{3}$, Gueriot ${ }^{4}$ and Conrad ${ }^{2}$ describe cases in which symptoms disappeared in the course of a few days. There is general agreement among these and other workers that pyuria tends to persist for a considerable time after the disappearance of symptoms.

Further progress.-Nine children died never having left hospital. Recovery was complete as measured by the disappearance of symptoms in all remaining fifty-two cases prior to their discharge. Contact was lost with four children after they left hospital. Forty-eight children were followed up in their homes and at the Royal Maternity Hospital Infant Clinic for periods varying from seven months to over two years: in no single instance was there a return of symptoms referable to the urinary tract: mental and physical progress was normal in all these children and the occurrence of neonatal urinary infection had not interfered with their later development.

\section{Fatal cases.}

Nine cases proved fatal and the following are their main clinical features together with autopsy findings when permission was obtained for postmortem examination. 
Case 2. Male (full-term, normal delivery). The baby showed a satisfactory condition for two days, on the third day refusing feeds, vomiting twice and not passing urine. On the fourth day there were sudden fever ( $101^{\circ}$ F.), colour changes, thirst, with later complete collapse. Urine was passed after 36 hours' interval (20 pus cells per high-power field), T. $101^{\circ} \mathrm{F}$. to $103 \cdot 8^{\circ} \mathrm{F}$. On the fifth day thirst, sweating, restlessness, pallor and collapse on two occasions occurred. T. $101^{\circ}$ F. $-102 \cdot 8^{\circ}$ F. He died in convulsions in the evening. No post-mortem examination was permitted.

Case 8. Male (twin, premature, weighing $2 \mathrm{lb} .12$ oz.). Born by Caesarian section for placenta praevia. Showed a moderately satisfactory condition for two days. On the third, fourth and fifth days there was occasional vomiting. On the sixth day occasional vomiting occurred, no urine was passed and suprapubic oedema was noted. On the eighth day, there was increased oedema with vomiting, T. $105^{\circ}$ F., marked bacilluria and pyuria (10 per high-power field) and thirst. On the ninth day no urine was passed. On the tenth day, T. $104 \cdot 8^{\circ} \mathrm{F}$, , urine was passed, pyuria (14 per high-power field) was noticed, the oedema was less and colour changes occurred. On the twelfth day, return of oedema and frequent green motions were noted leading to death. Post-mortem examination (48 hours after death) showed the kidneys congested at boundary zone. Microscopically there was extensive post-mortem autolysis, pelvis healthy.

Case 14. Female (full-term, high forceps delivery), was asphyxiated at birth, but there was no superficial bruising at site of forceps application. Symptoms of severe intracranial distress were present from second to fourth day, with convulsions and fever and two urinary examinations were negative. The cerebral condition settled by fifth day the child being weak and exhausted. On the seventh day, anuria, fever and occasional vomiting occurred. On the eighth day there was sweating and urine was passed once. On the ninth day, T. $100-105^{\circ} \mathrm{F}$., pyuria (25 per high-power field) and bacilluria, colour changes, oedema, great thirst and restlessness occurred. The condition remained unchanged with great fluctuations of temperature until thirteenth day when the child collapsed following a convulsion. Death occurred on fourteenth day. At autopsy, a tentorial tear and subdural haemorrhage were found with clotted blood in posterior fossae of base of skull. The kidneys showed intense congestion of the apices of pyramids, and microscopically, dilated collecting tubules, containing a number of polymorphonuclear leucocytes and infiltration of interstitium by polymorphonuclear leucocytes in localized patches.

Case 31. Male (full-term, normal delivery), showed a satisfactory condition for two days. On the third and fourth days he was restless with a whining cry, T. $98 \cdot 8^{\circ}-99 \cdot 4^{\circ} \mathrm{F}$. On the fifth dav, rapid respirations, marked pallor, extreme restlessness and great thirst were noted with T. $105^{\circ} \mathrm{F}$., and in the urine, 140 cells per high-power field, numerous casts and coliform organisms. On the sixth day, vomiting and frequent green motions were present and T. $103^{\circ}-105^{\circ}$ F. Otherwise there was no change. On the seventh to fifteenth day the condition persisted. The baby. became irritable and gradually weaker, with frequent colour changes, extreme thirst. The urinary findings were unchanged, 
but an extensive pustular skin condition developed. T. $101^{\circ}$ to $105^{\circ} \mathrm{F}$. Death took place on the fifteenth day. No postmortem examination was allowed.

Case 32. Male (premature, weighing $2 \mathrm{lb} .3 \mathrm{oz}$. , normal delivery). His frail condition was maintained during first three days. On the fourth day, he did not pass urine, T. $99^{\circ}$ F. There was suprapubic oedema, and he was refusing feeds. On the fifth to tenth day he passed small amounts of urine at long intervals, becoming restless, with great thirst, T. $98 \cdot 8^{\circ}-99 \cdot 4^{\circ} \mathrm{F}$. On the eleventh day, urine was passed once daily and there was pyuria (14 per high-power field), bacilluria, fretfulness, sweating and colour changes, T. $101^{\circ}$ F. On the twelfth to fifteenth day, he was getting weaker with severe turns of collapse and terminal green motions. Death occurred on fifteenth day. No postmortem examination was allowed.

Case 33. Female (full-term, difficult breech extraction with forceps to after-coming head). Facial paresis was noted. On the first day her condition was satisfactory and she passed urine. On the second day no urine was passed. The labia were swollen and the baby was fretful and restless. On the third day, urine was passed after 40 hours' interval and contained pus (12 per high-power field), a few red blood cells and numerous coliform organisms. Sweating and marked pallor were noted, T. $100^{\circ} \mathrm{F}$. On the fourth day she passed large amounts of urine, T. $99 \cdot 5^{\circ} \mathrm{F}$. The pyuria remained much the same, the oedema less and thirst and pallor persisted. From the fifth to eighth day the clinical condition improved and there was a diminution in pus cells in urine. On the eleventh day, T. $104 \cdot 6^{\circ}$ F., there was vomiting, an apprehensive expression, nystagmus, and the picture of intracranial distress. On the thirteenth day, the diagnosis of intracranial haemorrhage was confirmed by lumbar puncture findings; the urine still contained pus (10-15 per high-power field) and coliform organisms. Death took place on nineteenth day. Postmortem examination showed an intraventricular haemorrhage with gross destruction of brain substances. There was marked hyperaemia of the apices of the renal pyramids, uric acid deposits in straight tubules. Microscopically, the collecting tubules of kidney contained a number of pus cells and debris, uric acid deposits in the straight tubules, but no inflammatory infiltration and the pelvis was healthy.

Case 43. Male (full-term, normal delivery), had slight asphyxia at birth. During first two days his condition was satisfactory: he passed urine once in 48 hours. On the third day, blood stained vomiting occurred, and the baby became exhausted, with grey colour and diarrhoea. On the fifth day, vomiting of bright red blood occurred and no urine was passed for 48 hours. T. $100 \cdot 6^{\circ} \mathrm{F}$. There was some tenderness in left abdomen, with sweating and thirst. On the sixth day foetid motions were passed and only a small amount of urine (14 pus cells per high-power field, occasional red blood cells, bacilluria). Oedema and turns of collapse developed, but there was no sickness. T. 100-104.4 $\mathrm{F}$. On the seventh day, the condition was weaker with cyanotic turns, physical signs suggestive of pneumonia and occult blood in stools, T. $102-104 \cdot 8^{\circ} \mathrm{F}$. He gradually sank, with coarse twitching movements of extremities and small subcutaneous haemorrhage in cheek, T. $100-102^{\circ}$ F. Death occurred on ninth 
day. The post-mortem findings were a ' horse shoe' kidney, with some degree of hydronephrosis as result of pressure on the pelvis, and pneumonia. Microscopical examination of the kidney tissue showed structure developmentally normal, with extreme congestion at apex of pyramids, and haemorrhage into stroma. Many collecting tubules were dilated, containing debris, epithelial cells, occasional polymorphs. There were several small interstitial haemorrhages, but no interstitial inflammatory infiltration.

Case 46 (fig. 5). Female (normal delivery, premature, $5 \mathrm{lb} .0 \frac{1}{2} \mathrm{oz}$.). Her condition was satisfactory for two days. On the third day, no urine was passed, and she was thirsty, restless and jaundiced. On the fourth day, pyuria (50 pus cells per high-power field) was noted with numerous coliform bacilli, the urine was passed once in 36 hours. On the fifth day periods of grey pallor and two convulsions occurred with thirst and uric acid napkin deposits. T. $98 \cdot 0^{\circ}$ F. On the seventh day, T. $97 \cdot 2^{\circ}$ F., the baby was collapsed with definite anaemia. At post-mortem examination there were no definite findings beyond general anaemia, and congestion of kidneys with uric acid infarcts. Microscopical examination of kidney tissue showed intense congestion of tips of pyramids, great dilatation of ducts and dense, diffuse infiltration of interstitium by masses of polymorphs. Polymorphs present also in tubules. There was suppuration with necrosis and disappearnce of epithilium of pelvis with inflammatory cells in lumen.

Case 48. Male (full-term, normal delivery). His condition was excellent during first few days and weight was on the increase. On the sixth day, occasional vomiting was noted, T. 98-102.8 $8^{\circ} \mathrm{F}$. On the seventh day he was still vomiting with sweating, intense thirst, rapid respirations and collapse in evening. On the eighth day, loose offensive stools and frequent colour changes occurred, T. $99-100^{\circ}$ F. On the tenth day, no urine was passed for 48 hours and there was doubtful resistance to palpation in the right kidney angle. On the eleventh day, pyuria (45 cells per high-power field) was noted. T. $105^{\circ} \mathrm{F}$. He was passing large amounts of urine, otherwise there was no change. On the thirteenth day, urine was passed once in 48 hours, T. 100-102 $\mathrm{F}$. There were extreme colour changes and frequent vomiting, occasionally forcible with oedema and a convulsion in evening. On the fifteenth day, a septic skin and mouth condition was noted leading to death. At post-mortem examination the macroscopic findings were indefinite beyond partial atelectasis. Microscopic examination of kidney tissue showed groups of collecting tubules containing pus cells, the condition not being widespread and the areas affected small. There was no interstitial inflammatory infiltration but great congestion especially at apices of pyramids.

Six autopsies were carried out. Post-mortem degeneration of the tissues was so advanced in one of them that microscopic examination was valueless. The complete histological picture of early suppurative pyelonephritis was present in one case (no. 46). The findings in the remaining five cases were limited to the presence of pus cells in dilated collecting tubules and intense congestion at the apices of the pyramids: in addition, early leucocytic infiltration of the interstitium was present in one and interstitial haemorrhages in another. Uric acid infarcts were noted in two cases. Grossly abnormal development of the kidneys characterized one case. 
Discussing the findings in cases coming to autopsy with a clinical diagnosis of pyelitis Hoppe ${ }^{8}$ remarks upon the frequency with which pathological changes are entirely absent or are located in the kidney substance and not in the pelvis. He draws attention to the danger if autopsy is delayed of post-mortem autolysis rendering microscopical examination of kidney tissue valueless. Still ${ }^{14}$ is of the opinion that although kidney involvement is often present in fatal cases of pyelitis there is no associated nephritis in the majority of those that survive. Holt ${ }^{7}$ on the other hand considers that in severe cases some degree of pyelonephritis is present: this he contends may take the form of degeneration or only collections of either polymorphonuclear leucocytes or small abscesses in the renal parenchyma.

\section{Treatment.}

Breast feeding was employed where the child's condition remained good and provided there were no signs of collapse and that high fever was not present. Where breast milk was either not available or not desirable, feeds were of cow's milk, skimmed, sweetened and diluted. Extra fluids in the form of water and weak tea were given between feeds and in the presence of collapse or hyperpyrexia these fluids were given at hourly or half-hourly intervals and milk temporarily excluded. The tea, while helping to lessen the thirst, had a definite diuretic effect and possibly acted as a mild stimulant.

Alkalies were given in the form of sodium or potassium citrate. An initial dose of 16-20 grains in twenty-four hours was usually employed: in a number of cases this sufficed to alkalinize the urine but in others as many as 60-80 grains daily were required before the reaction of the urine changed. Dosages were then adjusted in order to maintain alkalinity. In several cases the clinical condition showed marked betterment and the temperature fell before the urine had become alkaline: in two cases recovery was complete although the urine never became alkaline. Among the cases followed up after their discharge from hospital a considerable number continued to pass acid urine containing pus and bacteria for a number of weeks without there being any return of symptoms.

Attention was paid to bowel function and regular evacuation aimed at: an initial dose of castor oil (30 minims) was given as a routine and where intestinal irritability was a feature small doses of a paraffin preparation were prescribed (e.g. Emuls. petrol. B.P. 30 minims three times a day). When the motions were foetid or contained mucus, bowel lavage was carried out daily.

In the severe cases local warmth was applied to the loins in the form of an antiphlogistine poultice and this appeared to have a distinctly beneficial effect on kidney excretion. Where colicky pain was present relief was afforded by carrying the poultice over the abdomen.

Tepid baths were given when there was maintained hyperpyrexia and small repeated doses of brandy used as a stimulant, 


\section{Discussion.}

The observations indicate the characteristic nature of the clinical picture in urinary disorders occurring in the newborn. There was a striking similarity in the signs and symptoms in all the cases. Infection of the urine was demonstrated in each instance but the microscopical abnormalities in the urine were varied. In several instances symptoms occurred before the appearance of abnormalities in the urine and in a large number of cases bacteruria and pyuria persisted for many weeks after the disappearance of symptoms. Symptoms were closely related to the excretory activity of the kidneys: aggravation of symptoms coincided with diminution in urinary output and improved with successful diuresis. It is possible that the initial occurrence of symptoms of general distress and of secondary urinary infection were contributed to by factors further limiting the small amount of concentrated urine normally associated with the early days of life.

In children suffering from intracranial birth injury, symptoms arising from urinary infection occurred later than in other cases: this late incidence may have been contributed to by the diminished urinary excretion resulting from lessened fluid intake and sedative treatment. The occurrence of urinary complications in these cases is not common. The nine cases described represent a small percentage of cases of cerebral birth injury seen during the period of these investigations. Apart from children with injuries of this sort the nature of the delivery did not appear to contribute towards the urinary condition: thirty-seven were spontaneously delivered, forceps were used in twelve and Caesarian section was performed in two.

The majority of the recorded cases of urinary infection in the newborn are in males, a fact which Conrad ${ }^{2}$ emphasizes when describing three cases in girls. The figures of this series, forty males and twenty-one females (or thirty-five and sixteen respectively, after eliminating cases complicated by an earlier cerebral condition), indicate that urinary disorders may occur in newborn children of either sex. Conrad ${ }^{2}$ suggests that infection is ' congenital' and in support of this quotes two instances of pyelitis in newborn children whose mothers suffered from the same condition during pregnancy. Such a theory does not completely explain the one instance in the present series of urinary infection in both mother and child, as in the former the organism was a streptococcus and in the latter b. coli. Infection of the urine by spread from the alimentary tract has always been considered as likely. In the present series grossly abnormal stools were passed in ten cases. In two of these the sequence of events consisted of a particularly severe stomatitis with a secondary involvement of the bowel followed by urinary infection: in the remaining eight cases urinary and alimentary disturbances occurred within a short period of one inother. These cases do no more than suggest the possibility of a common source of infection and in the remaining fifty-one cases of the series there was no clinical evidence pointing to the bowel as a source of the urinary infection. 
According to Addison ${ }^{1}$ congenital abnormalities account for a considerable number of cases of pyuria in older children but they contributed to only one fatal result in the present investigation (case 43). This was the only example of a developmental error of the urinary tract encountered in over two hundred consecutive post-mortem examinations on newborn infants. It is significant that among autopsies carried out on older subjects three children with hydro-ureter and hydronephrosis all died at the age of five months.

Post-mortem findings in the present investigation demonstrated that pathological changes were limited to the urinary tract (apart from the cerebral haemorrhage in cases 14 and 33), and with the exception of one case (46) were confined to the kidney substance. The changes were usually slight and it is justifiable to assume that they were even less so in surviving cases: the unlikelihood of there being any permanent morbid changes is borne out by the complete recovery noted in connection with all these cases.

The results obtained in the present series indicate that alkalies are as effective in the newborn as in older children and they also favour the view that the value of alkalies is their diuretic action. The chances of recovery are good. Prognosis must be guarded until satisfactory diuresis is attained, more particularly where there is persistent oedema, however slight. Convulsions are of grave significance and if associated with anuria are likely to be followed by death. The height of the fever is of little prognostic value: there may be no rise of temperature in even the most serious cases. The duration of the illness varies from one to many weeks and convalescence is frequently protracted. Recovery is usual and it is always complete.

\section{Summary.}

1. The abnormal microscopical and bacteriological findings in the urine of sixty-one newborn children with urinary disorders are described and details given of the clinical signs and symptoms. The urinary findings are compared with those in a series of healthy newborn children.

2. The abnormalities present in the urine were varied. Organisms were present in every case, pus was present in large amount in fifty-three cases : excessive deposits of uric acid and urates were noted in twenty cases, in five of which there was no pyuria.

3. A characteristic clinical picture is found in association with urinary conditions in the newborn. Onset of the illness is usually indefinite: prolonged anuria, especially when accompanied by fever or occasional vomiting is suggestive of a commencing urinary condition and justifies immediate diuretic treatment. Later restlessness, thirst and sweating develop and colour changes are characteristic of the illness at its height. Collapse occasionally occurs: high fever is common but a few cases run an afebrile course. Convulsions are of grave significance. 
4. Symptoms are closely related to the excretory activity of the kidneys and treatment should be directed towards the promotion and maintenance of adequate diuresis. This is best attained by the giving of fluids at frequent intervals and the administration of alkalies and weak tea.

5. Recovery is usual. A ' follow-up' of children after their discharge from hospital showed that recovery is complete, remissions rarely occur and the subsequent general health of the children is not impaired.

6. Urinary infection in the neonatal period occasionally occurs either as a complication of intracranial birth injury or in association with severe alimentary disturbance.

7. In fatal cases pathological changes are slight: they are most often found in the kidney substance and rarely involve the pelvis of the organ.

I should like to express my thanks to Professor Charles McNeil for valuable criticism offered during the preparation of this paper, to Dr. Agnes R. MacGregor for bacteriological and histological opinions, and to Dr. T. Y. Finlay for assistance in following up a number of children after discharge from hospital.

\section{REFERENGES.}

1. Addison, D. L., Brit. Med. J., Lond., 1932, ii, 969.

2. Conrad, C. E., Am. J. Dis. Child., Chicago, 1926, XXXI, 253.

3. Graham, R. H., Am. J. Med. Sc., Philad., 1925, CLXX, 401.

4. Gueriot, P., Bull. Soc. d'obst. et de gynec, Paris, 1934, XXIII, 42.

5. Helmholz, H. F., Abt's. Pediatrics, 1924, IV, 923.

6. Helmholz, H. F., \& Millikin, F., Am. J. Dis. Child., Chicago, 1922, XXIII, 309.

7. Holt, E. L., \& Howland, J., Dis. of Infancy and Childh., New York, 1926, 515.

8. Hoppe, L. D., Arch. Pediat., New York, 1924, XLI, 31.

9. Litchfield, H. R., \& Gillman, J. H., ibid., 1932, XLIX, 776.

10. Paterson, D., Lancet, Lond., 1931, ii, 1187.

11. Ramsay, W. R., Am. J. Dis. Child., Chicago, 1922, XXIV, 218.

12. von Reuss, A. R., Dis. of the Nere Born, Lond., 1920.

13. Sauer, L. W., J. Am. Med. Ass., Chicago, 1925, LXXXV, 327.

14. Still, G. F., Common Disorders and Dis. of Childh., London, 1927.

15. Thomson, J., \& Findlay, L., Clinical Study and Treat. of Sick Child., Edinburgh, 1933, 434. 